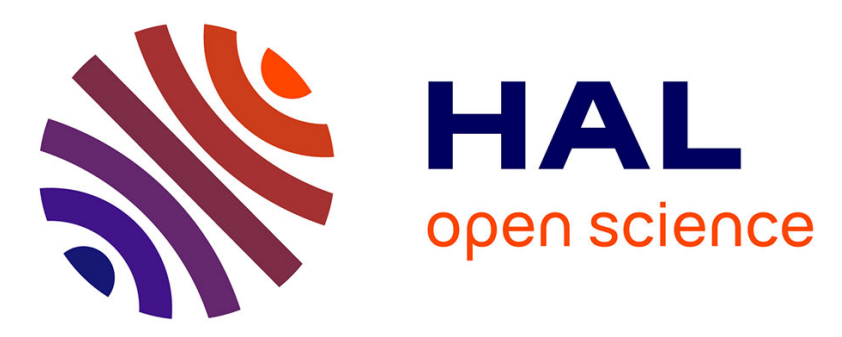

\title{
Details of the Crystalline Ultrastructure of C-Starch Granules Revealed by Synchrotron Microfocus Mapping
}

Alain Buleon, Catherine Gérard, Christian Riekel, Roger Vuong, Henry

Chanzy

\section{- To cite this version:}

Alain Buleon, Catherine Gérard, Christian Riekel, Roger Vuong, Henry Chanzy. Details of the Crystalline Ultrastructure of C-Starch Granules Revealed by Synchrotron Microfocus Mapping. Macromolecules, 1998, 31 (19), pp.6605-6610. hal-02687400

\section{HAL Id: hal-02687400 \\ https: / hal.inrae.fr/hal-02687400}

Submitted on 1 Jun 2020

HAL is a multi-disciplinary open access archive for the deposit and dissemination of scientific research documents, whether they are published or not. The documents may come from teaching and research institutions in France or abroad, or from public or private research centers.
L'archive ouverte pluridisciplinaire HAL, est destinée au dépôt et à la diffusion de documents scientifiques de niveau recherche, publiés ou non, émanant des établissements d'enseignement et de recherche français ou étrangers, des laboratoires publics ou privés.

$$
\text { Copyright }
$$




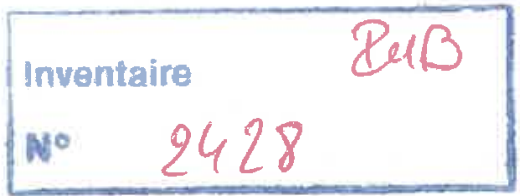

\section{Details of the Crystalline Ultrastructure of C-Starch Granules Revealed by Synchrotron Microfocus Mapping}

\section{Alain Buléon, Catherine Gérard, Christian Riekel,} Roger Vuong, and Henri Chanzy

Institut National de la Recherche Agronomique, B. P. 71627, 44316 Nantes Cedex 3, France, European Synchrotron Radiation Facility, BP 220, 38043 Grenoble Cedex, France, and Centre de Recherches sur les Macromolécules Végétales, CNRS, BP 53, 38041 Grenoble Cedex 9, France, affiliated with the Joseph Fourier University of Grenoble, France

Reprinted from

Volume 31, Number 19, Pages 6605-6610 


\title{
Details of the Crystalline Ultrastructure of C-Starch Granules Revealed by Synchrotron Microfocus Mapping
}

\author{
Alain Buléon, ${ }^{\dagger}$ Catherine Gérard, ${ }^{\dagger}$ Christian Riekel, ${ }^{\ddagger}$ Roger Vuong, ${ }^{\S}$ and \\ Henri Chanzy*,8
}

Institut National de la Recherche Agronomique, B. P. 71627, 44316 Nantes Cedex 3, France, European Synchrotron Radiation Facility, BP 220, 38043 Grenoble Cedex, France, and Centre de Recherches sur les Macromolécules Végétales, CNRS, BP 53, 38041 Grenoble Cedex 9, France, affiliated with the

Joseph Fourier University of Grenoble, France

Received May 8, 1998; Revised Manuscript Received July 20, 1998

\begin{abstract}
A microfocus synchrotron wide-angle diffraction mapping experiment was used to analyze the crystalline microstructure and the polymorphism of a series of C-starch granules from smooth pea. In these specimens that globally contained $60 \%$ of A-phase and $40 \%$ of the B-allomorph, it was found that the two phases were present in each granule. The A-allomorph was located essentially in the outer part of the granules whereas the B-phase was found mostly at their center. In the A-component, the diffraction diagrams were always poorly oriented fiber patterns with the fiber axis systematically oriented toward the center of the granule. On the other hand, in the B-center, only powder diagrams could be observed whereas in middle zones of the granules, the B-component was much better oriented than the A-component.
\end{abstract}

\section{Introduction}

The crystalline nature of starch has been established for many decades, ${ }^{1,2}$ and on the basis of their X-ray diffraction patterns, the various starch specimens can be classified into three main categories namely $\mathrm{A}, \mathrm{B}$, and C. While A-starch is found chiefly in cereals, B-starch occurs in most tubers. C-starch, which is less frequent, is found in some legumes. ${ }^{3-6}$ In terms of structural work, a number of reports have dealt with the elucidation of the molecular and crystal structure of A-and B-starches. ${ }^{7-10}$ In these two allomorphs, it is believed that it is the short amylose terminal segments of the amylopectin molecules that constitute the crystalline domains. In A- and B-starches, these segments are thought to have the same double helical conformation but different packing arrangement and different intracrystalline water content. The case of $\mathrm{C}$-starch is more complex as its X-ray diffraction diagram can be resolved as a combination of A- and B-crystalline constituents, the amount of each of these depending on the sample origin.

The exact location of the A- and B-components in C-starch has been debated for a long time. In fact the granules of C-starch, as all those of the common starches, are far too small to be studied individually by conventional X-ray diffraction techniques. For these samples, up to very recently, one had access only to $\mathrm{X}$-ray powder diagrams that average out a large number of granules in all possible orientations. Given these experimental constraints, it has not been possible so far to use diffraction techniques to decide whether in C-starches the two phases A and B were found in the same granules or whether there were granules that were pure $A$ and others that were pure $B$.

Quite recently, in a study on smooth pea starch-the best known C-starch specimen-it was shown that these

* Corresponding author.

$\dagger$ Institut National de la Recherche Agronomique.

‡ European Synchrotron Radiation Facility.

\& Centre de Recherches sur les Macromolécules Végétales. granules dispersed into dilute aqueous $\mathrm{KCl}$ underwent a stepwise gelatinization when the temperature of the gelatinizing medium was increased..$^{11}$ At around $65^{\circ} \mathrm{C}$ only the inner part of the granule became swollen whereas the outer part remained intact until around $75{ }^{\circ} \mathrm{C}$ where this part started also to gelatinize. By comparing this behavior with that of standard A- and B-starches, the authors of this study concluded that the center of the granules consisted mainly of the Ballomorph whereas the A-phase was located toward the outside of the granules.

In the present work, the localization of the polymorphic crystalline components of C-starch was investigated by synchrotron microfocus mapping. This wide-angle diffraction technique, developed recently at the European Synchrotron Research Facility (ESRF) has allowed to map out the local crystalline orientation and the crystallinity of individual starch grains with a current resolution of around $2-3 \mu \mathrm{m} .{ }^{12-13}$ In this paper, the method was used for C-starch from smooth pea with the goals of (i) locating the A- and B-phases in the C-starch granules and (ii) identifying the orientation of the crystalline domains within these granules.

\section{Experimental Section}

Sample Preparation. A sample of smooth pea from Grinsted, batch no. 011509 was used throughout this work. The granules which had a fairly narrow distribution in size had an average diameter of $29 \mu \mathrm{m}$. A spatula tip of this sample was dispersed into $10 \mathrm{~mL}$ of a dilute aqueous solution of poly(acrylic acid) $(0.15 \%, w / v)$. Drops of this suspension were deposited on collodion coated $3 \mathrm{~mm}$ electron microscope grids (copper Pelco slot grids, with a slot of $0.4 \times 2 \mathrm{~mm}$ ). Once dried, these grids were observed with a Zeiss Axiophot optical microscope, and photomicrographs showing the location of the starch granules were made, under both polarized light and Nomarski contrast. These grids were stored until further use in a desiccator where a saturated solution of $\mathrm{NaBr}$ maintained a constant humidity atmosphere (relative humidity $\mathrm{RH}=$ $75 \%$ ).

Synchrotron Experiments. Experiments were performed at the microfocus beam line (ID13) at the European Synchro- 


$$
\begin{aligned}
& \text { foer ant } \\
& \text { III I III } \\
& \text { (")" "य) }
\end{aligned}
$$$$
\text { 'III, III) }
$$

A STARCH

B STARCH

Figure 1. Schematic fiber diffraction pattern with vertical fiber axis of A- and B-starch.

tron Radiation Facility (ESRF). The set up consisted of a $\mathrm{Si}_{111}$ monochromator, an ellipsoidal mirror, and a tapered glass capillary producing a monochromatic X-ray beam $(\lambda=0.092$ $\mathrm{nm}$ ) of $2 \mu \mathrm{m}$ full width at half-height at the exit of the capillary. A $10 \mu \mathrm{m}$ platinum aperture, positioned at the exit of the capillary, was used to reduce the background from the optics. The grids holding the starch granules were mounted at the eucentric position of a goniometer head positioned on a motorized $\mathrm{x} / \mathrm{y}$ stage with submicrometer positional resolution. The grids holding the sample were positioned at about $0.5 \mathrm{~mm}$ from the exit of the capillary with the result of a slight increase of the tails of the beam profile, as determined by a knife-edge scan. At the sample level, the flux density was $\leq 10^{8}$ photons/ $\mathrm{s} / \mu \mathrm{m}^{2} / \mathrm{mA}$. The recordings were made with an image-intensified CCD camera with video-readout and on-line digitizer, positioned at about $50 \mathrm{~mm}$ from the samples. Starch granules were initially observed on the sample stage outside of the X-ray beam with the help of an optical microscope hooked to a video system. A specific granule was then transferred into the X-ray beam with a precision of a few $\mu \mathrm{m}$. Diffraction diagrams were usually recorded by a mesh scan with $7 \mu \mathrm{m}$ steps, centered on the granule with a recording time of $16 \mathrm{~s} / \mathrm{image}$ corresponding to the sum of 400 video frames. At the end of the experiments, photomicrographs in Nomarski contrast of the sample grids revealed the pattern of irradiation of the sample. The series of diffraction patterns were then processed with the program FIT $2 D^{14}$ and their contrasts optimized with the program NIH Image.

X-ray Analysis of A and B Composition. A sample of smooth pea starch ( $20 \mathrm{mg}$ dry matter, $\% \mathrm{H}_{2} \mathrm{O}=28$ ) was sealed between two Kapton foils, to prevent any significant change in water content during the measurement. Transmission diffraction diagrams were recorded with a XRG 3000 X-ray generator (Inel Orléans-France) operated at $40 \mathrm{kV}$ and $30 \mathrm{~mA}$ fitted with a quartz monochromator yielding $\mathrm{Cu} \mathrm{K} \alpha_{1}$ radiation $(\lambda=0.15405 \mathrm{~nm}$ ). A curved position sensitive detector (Inel CPS120) was used to monitor the diffracted intensities using $3 \mathrm{~h}$ exposure periods. The respective amounts of $\mathrm{A}$ - and B-types were determined by applying a multilinear regression, assuming that the experimental normalized diagrams were a linear combination of elementary patterns of amorphous together with A- and B-types, following a method derived from Gernat et al. ${ }^{15}$ While recrystallized amyloses were used for A- and B-crystalline standards, extruded potato starch was used as an amorphous reference. Diffraction diagrams of pea starch and standards were all normalized at the same total scattering value, with $2 \theta$ ranging from 3 to $30^{\circ}$.

\section{Results and Discussion}

A survey of the reported fiber diffraction patterns of A- and B-starch indicates that the two patterns have some similarity with nearly the same fiber repeat and a number of strong diffraction spots occurring at nearly the same position in the diagram. The two patterns are illustrated schematically in Figure 1. Despite their similarity, some strong diffraction spots can be selected as characteristics of A- and B-starch. Typically, along the equator, the strong reflection at a $d$ spacing of 1.61 $\mathrm{nm}\left(2 \theta=5.5^{\circ}\right.$ for a Cu $\mathrm{K} \alpha_{1}$ wavelength of $\left.0.15405 \mathrm{~nm}\right)$ is specific for B-starch whereas a reflection at a $d$ spacing of $0.491 \mathrm{~nm}\left(2 \theta=18^{\circ}\right)$ can be attributed to A-starch only. In the first layer line, a diffraction spot at $0.487 \mathrm{~nm}$, absent in B-starch, is also characteristic

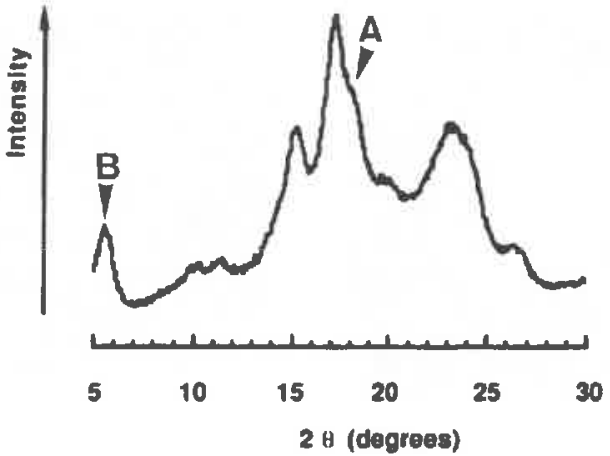

Figure 2. Powder X-ray trace of a sample of smooth pea starch. The diffraction peak at $2 \theta=5.5^{\circ}$ is typical of the B-phase whereas the hump at $2 \theta=18^{\circ}$ (arrowed) is the signature of the A-phase.

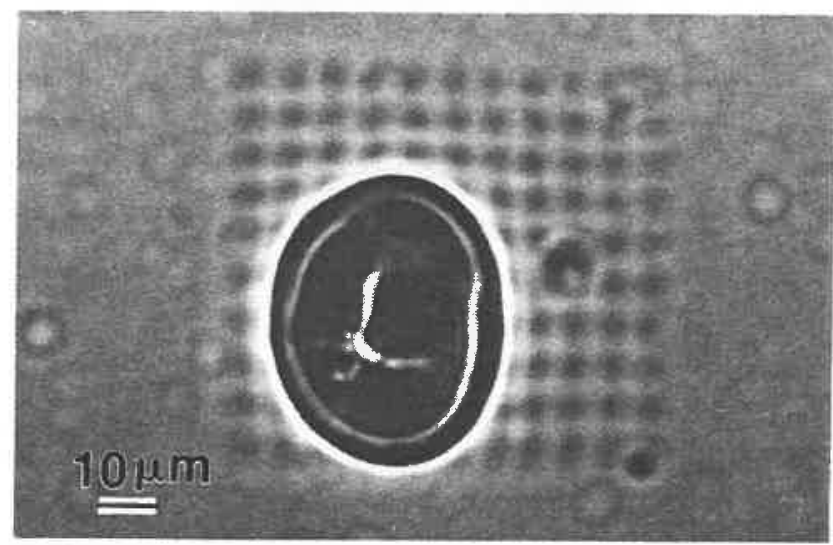

Figure 3. Optical micrograph in Nomarski contrast of a typical sample of smooth pea after a $7 \mu \mathrm{m}$ step irradiation with an about $2 \mu \mathrm{m}$ X-ray beam, each step consisting of a $16 \mathrm{~s}$ exposure. The imprint of the beam is associated with the beam damage in the supporting collodion film.

of A-starch. Thus, for poorly oriented samples, a broad arc located at around $0.49 \mathrm{~nm}$ and extending almost to the second layer line can be considered as the signature of the A-allomorph.

The powder X-ray trace of the sample of smooth pea starch that was used here is shown in Figure 2. This trace reveals the main crystalline features of $\mathrm{C}$-starch with a diffraction peak at $5.5^{\circ}(d=1.61 \mathrm{~nm})$ that is the indication of B-starch and a composite series of peaks at around $17-18^{\circ}$ with a clear hump at $18^{\circ}(d=0.491$ $\mathrm{nm}$ ) corresponding to A-starch. The deconvolution of this diagram indicates that the sample consisted of 60 $\pm 5 \%$ of A-starch and $40 \pm 5 \%$ of B-starch. These values are very similar to those already reported in the literature for such specimens. ${ }^{15,16}$

The analysis of individual smooth peas starch granules by microfocus synchrotron mapping is illustrated in Figure 3 which shows one of these grain and the 7 $\mu \mathrm{m}$ grid of synchrotron impacts on the supporting plastic film. Such impacts are not seen on the granule itself as it seems to be too thick for the Nomarski contrast that was used for the recording of Figure 3. Each exposure on the granule or on its edge gave a diffraction diagram showing some diffraction arcs or circles whereas those outside yielded only noisy background. As opposed to X-ray powder patterns of smooth pea starch, each diagram was different from the next one, either in composition or/and orientation. The diagrams could be classified into three families that are shown in Figure 4. In Figure 4a, located essentially in the outer part of 

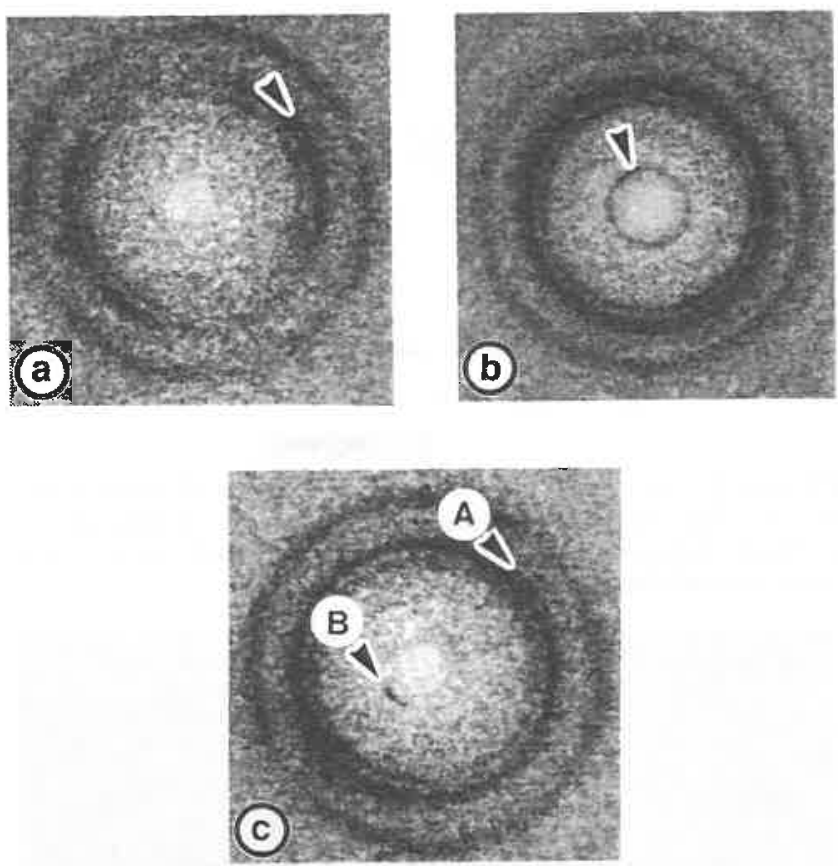

Figure 4. Typical transmission microfocus X-ray patterns recorded on smooth pea starch granules. (a) A fiber diagram identified as corresponding to the A-phase and taken in the outer layer of the granules. The arrow points toward the ring corresponding to a $d$ spacing of $0.491 \mathrm{~nm}\left(2 \theta=18^{\circ}\right)$ that is found only in the A-phase. (b) A powder diagram corresponding essentially to the B-phase and taken in the center of the granule. The arrow points toward the ring corresponding to a $d$-spacing of $1.61 \mathrm{~nm}\left(2 \theta=5.5^{\circ}\right)$ that is found only in the B-phase. In this diagram a small amount of A-phase can be detected, likely due to diffraction from the part of outer layer that surrounds the center of the granule. (c) A poorly oriented fiber diagram corresponding to intermediate zones of the granule and showing a classical C-starch type of pattern. The arrows 1 and 2 correspond respectively to diffraction arcs identified as corresponding to the A- and B-phases, respectively.

the granules, one had a typical A-starch pattern clearly identified by the arrowed diffraction ring, at $d=0.49$ $\mathrm{nm}$ which is unique in A-starch. In addition, the pattern in Figure $4 \mathrm{a}$ does not show any diffraction spot at $d=1.61 \mathrm{~nm}$ indicating that no B-phase could be detected. The patterns such as the one shown in Figure $4 \mathrm{~b}$ were persistently recorded in the central part of the granules. These patterns are rich in B-phase: they show clearly the inner ring at $d=1.61 \mathrm{~nm}$ (arrow in Figure 4b) but only a weak arc at $d=0.49 \mathrm{~nm}$ indicating a small amount of A-phase. Intermediate patterns containing mixture of $\mathrm{A}$ - and B-phases were also recorded from area intermediate between the center and the edge of the granules. One example of these is shown in Figure 4c where the arrow $A$ indicates clearly the presence of A-phase, whereas the arrow B corresponds to the B-phase signature. A pattern such as that in Figure 4c matches closely a theoretical diagram of C-starch drawn from the literature data (Figure 5). It corresponds also roughly to the powder pattern shown in Figure 2.

In Figure 6 is shown a grid of 18 patterns recorded every $7 \mu \mathrm{m}$ on one granule of smooth pea starch. With the exception of the upper left and upper right diagrams, which obviously were taken outside of the grain, all diagrams presented some diffraction information. A survey of these diagrams revealed clearly that in the same smooth pea starch granule, one could detect at

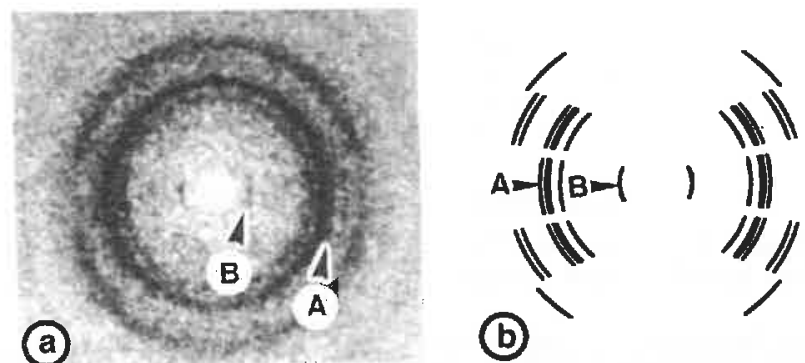

Figure 5. Observed (a) and theoretical (b) fiber diagrams of C-starch. Arrows 1 and 2 correspond to specific diffraction ares that are the signature of A- and B-starch, respectively.

the same time some A- and some B-components. In the 6 central patterns the B-phase appears to be dominating. In fact only a minor quantity of A-starch trace could be observed in these patterns which therefore must correspond to much more B-phase than the average of $40 \%$ that was derived from the global $X$-ray analysis. In addition these central diagrams appeared unoriented. In the outer part of the granules, the diagrams clearly denote a strong presence of A-starch. These diagrams are in fact poorly oriented fiber diagrams, with the fiber axis pointing systematically toward the center of the granule. The presence of a small amount of B-starch can be observed in some of these outer part patterns, but not in others, and it is difficult to decide whether minute quantities of B-starch can be found in the periphery of the granule.

From the results presented for the granule investigated in Figure 6 as well as for a number of other granules not described here, one can generate a composition map for smooth pea starch granules. This map, shown in Figure 7 indicates that, in these grains, the outer part is essentially composed of A-starch. On the other hand, the central part of the granules is predominantly composed of B-starch with only a little A-phase. This minor quantity of A-phase in the middle of B-phase may be genuine. However it is more likely due to the fact that before hitting the center of the granules, the impinging $X$-ray beam must first cross some of the outer part of the granule that is in A-phase. Similarly it hits also some of this phase on the other side of the granule. These two layers of A-phase may be responsible for the small amount of A-allomorph detected in the center of the granules. The situation is different with the X-ray diagrams taken on the outer part of the granules where only this part is exposed to the X-ray beam.

The observations presented in this study confirm the recent results of Bogracheva et al. ${ }^{11}$ who reported also that smooth pea starch granules had their central part in B-allomorph and their outer part in A-type. In their method, they followed the loss of birefringence of the granules during gelatinization in aqueous $\mathrm{KCl}$ solution. By slowly raising the gelatinization temperature, they could observe that, at temperatures up to around $65^{\circ} \mathrm{C}$, only the inner part of the granules lost their birefringence. For the outer parts, this loss required a temperature of up to $75^{\circ} \mathrm{C}$. By analogy with test samples of pure A- and B-starches, they could draw conclusions about the dual morphology of the smooth pea granules. We think that our method is a more direct method to identify the location of the two crystalline phases of C-starch. Indeed, the observation of the loss of birefringence that was used by Bogracheva et al. ${ }^{11}$ is not necessarily equivalent to a loss in crystallinity, but rather to a loss of chain orientation. It could very well 

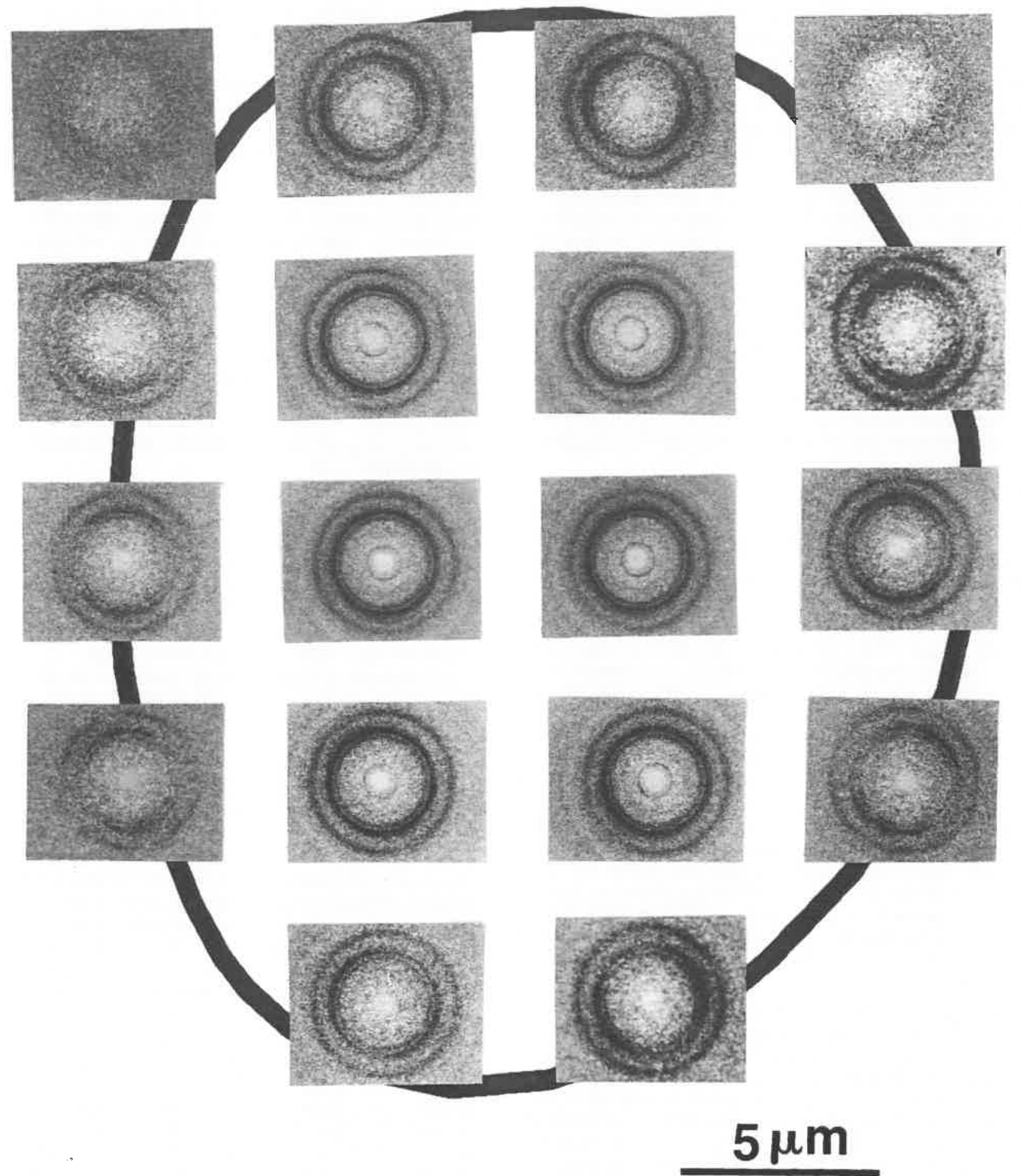

Figure 6. Set of microfocus X-ray diffraction patterns recorded on a starch granule of smooth pea. Each diagram corresponds to a diffraction area of about $3 \mu \mathrm{m}^{2}$ of the specimen and steps of $7 \mu \mathrm{m}$ separate the diagrams.

be that some crystallinity remains in some part of the granules even when the birefringence is totally lost. Conversely, the granules could still be birefringent, even when they are completely decrystallized. For these reasons, we plan to use also our technique to follow the gelatinization process of individual C-starch granules.

In the experiments that were done here, the specimens were embedded in poly(acrylic acid) and kept under 75\% RH until mounting in the synchrotron beam. We believe that our operating conditions where the samples may undergo a slight dehydration-limited by the poly(acrylic acid) barrier-are not too detrimental

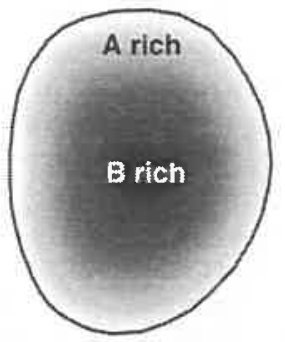

Figure 7. Schematic drawing of a C-starch granule of smooth pea showing the two zones: the center zone rich in B-type and the outer zone consisting of A-starch. 
to the success of the experiments. Indeed, the diffraction line at $d=1.61$ in the B-part of the diagram is most sensitive to the water content of the sample. ${ }^{17,18}$ If substantial dehydration had taken place, this diffraction line should be absent or drastically reduced. Since it is observed with a rather strong intensity, we deduce that the water content of the granules has remained fairly constant, and for this reason, the qualitative results of our observations appears to be quite valid. The A-part of the sample is less susceptible to dehydration as the intracrystalline water content is less in A-starch than in B-starch. In previous synchrotron experiments on potato (B-allomorph) and wheat (Aallomorph) starches, ${ }^{12}$ we could obtain well-resolved diffraction patterns of both samples even after several hours in the beam line environment. Thus, we believe that our results on the localization of the $A$ - and B-phases in smooth pea starches are essentially correct. In future work we plan to use an environmental system similar to or even better than the one used by Waigh et al. ${ }^{13}$

When comparing the microfocus diffraction diagrams obtained in the present work with those obtained on potato starch, ${ }^{12,13}$ one is struck by the rather poor radial orientation of the A-phase, even in the periphery of the smooth pea granules. On the other hand, as seen in Figure 4c, the B-phases appear to be much better oriented in intermediate zones between the periphery and the center of the granules. As a poor orientation was also systematically observed in the microfocus mapping of wheat starch granules that consist of A-starch, one can speculate that a poor radial orientation of the amylose segments may be one of the features of A-starch. On the other hand, the B-phase either in potato or in the present smooth pea starches would occur with a much better radial orientation except at the center of the granules where there is always a lack of orientation. These observations are only preliminary and are insufficient to draw general rules for the perfection of the orientation of the amylose segments in A- as opposed to B-starches. Further work with series of starch samples from different origin is underway to ascertain the generalization of the present observations.

The method that we have presented here could be improved further in several ways. In one of these, the X-ray mapping could be applied to micrometer-thick sections. This should allow to prove or disprove whether a small amount of A-phase is mixed with some B-starch in the center of the granules. Also, the orientation of the B-crystals in the center of the granules could be assessed. At present, the X-ray diagrams recorded in the center of the granules are of the powder type, likely because of the radial orientation of the crystals. This could be proven by an X-ray mapping analysis of contiguous sections resulting from serial sectioning. Another improvement to the present experiment would be to record the diffraction diagrams at liquid nitrogen temperature. It was indeed shown that, for biopolymers, such cooling could improve the lifetime of the specimen in the beam by an order of magnitude. Here we used a recording time of $16 \mathrm{~s}$ that corresponds more or less to the maximum recording time under the flux density of $\leq 10^{8}$ photons $/ \mathrm{s} / \mu \mathrm{m}^{2} / \mathrm{mA}$ before the total destruction of the sample. This recording time could therefore be vastly increased by using a cooling device leading to better X-ray patterns with substantially improved signal-to-noise ratio. ${ }^{19}$
The synchrotron microbeam that was used in the present study had a diameter of around $2 \mu \mathrm{m}$. This beam size is small enough to describe the gross ultrastructure of individual starch granules, and this is a substantial progress for the knowledge of structure of starch. This beam size is however too large to assess the details of the crystalline micromorphology of the individual concentric layers that are found within the granules. Indeed, as these layers are only around $\mathbf{5 0 0}$ $\mathrm{nm}$ in thickness, it means that, during the scanning of the granule, several of them will be diffracting at any given time. Thus in the present work, it is impossible to decide whether the A- and B-phases are present within the same single layer of the granule or along a single cluster of amylopectin. ${ }^{20}$ If this cannot be resolved today, one can nevertheless foresee future improvements with the ongoing development of synchrotron X-ray beams with submicrometer dimensions. ${ }^{21}$ Such a beam, when available, should be able to push the knowledge of the crystalline morphology of starch to the single layer level.

In the world of starch, one may wonder why most cereal starch granules contain the A-phase, those of tubers the B-phase, whereas some of the legume starches contain the mixed phase $A+B$ as observed here. This diversity has to be traced to the biocrystallization scheme that occurs during starch biosynthesis, but unfortunately, such a mechanism has not been elucidated so far. The determination of the crystalline polymorphism of starch is under genetic control through either the average chain length of the short amylose terminal segments of amylopectin or the branching scheme itself of the amylopectin molecules. At present, it is generally accepted that $\mathbf{A}$-starches have terminal amylose segments that are shorter than those of the B-types and that the C-starch terminal segment have intermediate lengths. ${ }^{22,23}$ Other factors that may play a role for the determination of the $A / B$ composition in starch are the temperature and hydration conditions. Indeed, during in vitro crystallization experiments of short amylose chains it was shown that the B-type allomorph was favored over the A- either at low crystallization temperature or at high water content. ${ }^{24,25}$ Along this line, it has been recently observed also that maize starch, which under normal weather conditions was of the A-type may contain up to $25 \%$ of the B-allomorph when the same plants are grown under wet and/or cold conditions. ${ }^{26}$ For the pea starches, it is almost impossible to analyze and compare the endsegment lengths of the amylopectin molecules located in the center with those at the granule periphery. Nevertheless, it could be conceived that the center of the granules could be biosynthesized under wetter conditions than the outer parts. The decrease in water content (from about $80 \%$ to about $45 \%$ ) in the pea hull during the maturation of the pea grains is well-known and is commonly used for the evaluation of the maturity of the pea grains. ${ }^{27}$ Despite these observations, the growth of pea starch granules has not been studied in detail, and it is not known whether the growth of these starch granules is concomitant with the grain maturation. Thus, it is not possible at the moment to explain without ambiguity the reason for the observed dimorphism of C-starch.

Acknowledgment. The authors thank B. Pontoire for technical assistance in this work. 


\section{References and Notes}

(1) Katz, J. R. Z. Physik. Chem., A 1930, 150, 37-59.

(2) Katz, J. R.; van Itallie, T. B. Z. Physik. Chem., A 1930, 150, 90-99.

(3) Sarko, A.; Wu, H.-C. H. Starch/Störke 1978, 30, 73-78.

(4) Blanshard, J. M. V. In Starch: Properties and Potential; Galliard, T., Ed.; Critical Reports on Applied Chemistry 13; Wiley and Sons: New York, 1987; pp 16-54.

(5) Gernat, Ch.; Radosta, S.; Damaschun, G.; Schierbaum, F. Starch/Stärke 1990, 42, 175-178.

(6) Guilbot, A.; Mercier, C. In The Polysaccharides; Aspinall, G. O., Ed.; Academic Press: Orlando, FL, 1985; Vol. 3, pp 209282.

(7) Wu, H.-C. H.; Sarko, A. Carbohydr. Res. 1978, 61, 7-25.

(8) Wu, H.-C. H.; Sarko, A. Carbohydr. Res. 1978, 61, 27-40.

(9) Imberty, A.; Chanzy, H.; Pérez, S.; Buléon, A.; Tran, V. J. Mol. Biol. 1988, 201, 365-378.

(10) Imberty, A.; Pérez, S. Biopolymers 1988, 27, 1205-1221.

(11) Bogracheva, T. Ya.; Morris, V. J.; Ring, S. G.; Hedley, C. L. Biopolymers 1998, 45, 323-332.

(12) Buléon, A.; Pontoire, B., Riekel, C.; Chanzy, H.; Helbert, W.; Vuong, R. Macromolecules 1997, 30, 3952-3954.

(13) Waigh, T. A.; Hopkinson, I.; Donald, A. M.; Butler, M. F.; Heidelbach, F.; Riekel, C. Macromolecules 1997, 30, 38133820.

(14) Hammersley, A. FIT2D software code; website: http://www.esrf.fr/computing/expg.
(15) Gernat, Ch.; Radosta, S.; Anger, H.; Damaschun, G. Starch / Stärke 1993, 45, 309-314.

(16) Cairns, P.; Bogracheva, T. Ya.; Ring, S. G.; Hedley, C. L.; Morris, V. J. Carbohydr. Polym. 1997, 32, 275-282.

(17) Buléon, A.; Bizot, H.; Delage, M. M.; Multon, J.-L. Starch/ Stärke 1982, 34, 361-366.

(18) Buléon, A.; Bizot, H.; Delage, M. M.; Pontoire, B. Carbohydr. Polym. 1987, 7, 461-482.

(19) Bram, A.; Bränden, C. I.; Craig, C.; Snigireva, I.; Riekel, C. J. Appl. Crystallogr. 1997. 30, 390-392.

(20) French, D In Starch Chemistry and Technology; Whistler, R. L., Bemiller, J. N., Paschall, J. F., Eds.; Academic Press: Orlando, FL, 1984; pp 183-247.

(21) Cedola, A.; Lagomarsino, S.; Di Fonzo, S.; Jark, W.; Riekel, C.; Deschamp, P. J. Synchrotron Radiat. 1998, 5, 17-22.

(22) Hizukuri, S.; Kaneko, T.; Takeda, Y. Biochim. Biophys. Acta, 1983, 760, 188-191.

(23) Gidley, M. J. Carbohydr. Res. 1987, 161, 301-304.

(24) Gidley, M. J.; Bulpin, P. V. Carbohydr. Res. 1987, 161, 291300.

(25) Buléon, A.; Duprat, F.; Booy, F. P.; Chanzy, H. Carbohydr. Polym. 1984, 4, 161-173.

(26) Buléon, A.; Colonna, P.; Planchot, V. Personal communication.

(27) Le Deunff, Y.; Rachidian, Z. J. Exp. Bot. 1988, 39, 1221-1230. MA980739H 
